\title{
Cases of Impaired Oxidative Burst in HIV-Exposed Uninfected Infants' Neutrophils-A Pilot Study
}

\author{
Anicet Christel Maloupazoa Siawaya ${ }^{1 \dagger}$, Amandine Mveang-Nzoghe ${ }^{1 \dagger}$, \\ Ofilia Mvoundza Ndjindji't, Armel Mintsa Ndong ${ }^{2}$, Paulin N. Essone ${ }^{1,3}$ and \\ Joel Fleury Djoba Siawaya ${ }^{\text {*t }}$

\begin{abstract}
'Unités de Recherche et de Diagnostics Spécialisés, Laboratoire National de Santé Publique, Libreville, Gabon, ${ }^{2}$ Unité de Virologie, Laboratoire National de Santé Publique, Libreville, Gabon, ${ }^{3}$ Faculty of Health Sciences, Institute of Infectious Diseases and Molecular Medicine (IDM), Division of Immunology and South African Medical Research Council (SAMRC) Immunology of Infectious Diseases, University of Cape Town, Cape Town, South Africa
\end{abstract}

OPEN ACCESS

Edited by:

Charles R. Rinaldo,

University of Pittsburgh, USA

Reviewed by:

Diana Marcela Giraldo,

Universidad de Antioquia, Colombia

Seema Desai,

Rush University, USA

*Correspondence:

Joel Fleury Djoba Siawaya

joel.djoba@gmail.com

tThese authors have contributed equally to this work.

Specialty section:

This article was submitted to HIV and AIDS,

a section of the journal

Frontiers in Immunology

Received: 23 August 2016

Accepted: 23 February 2017

Published: 09 March 2017

Citation:

Maloupazoa Siawaya AC, MveangNzoghe A, Mvoundza Ndjindji $O$, Mintsa Ndong A, Essone PN and Djoba Siawaya JF (2017) Cases of Impaired Oxidative Burst in HIV-Exposed Uninfected Infants' Neutrophils - A Pilot Study.

Front. Immunol. 8:262. doi: 10.3389/fimmu.2017.00262
An increased risk of serious bacterial infections in HIV-exposed uninfected (HEU) infants has been demonstrated. Although neutrophils are essential for the protection of infants against bacterial infections, no study has investigated their profile in HEU infants to date. In this study, we assessed the function of neutrophils in HEU infants using the nitroblue tetrazolium reduction test. Among $25 \mathrm{HEU}$ infants, 9 (36\%) showed a reduced ability of their neutrophils to produce reactive oxygen species upon stimulation with bacteria. No alteration of total neutrophil counts was noted in the blood of HEU infants indicating that the alteration observed in the $36 \%$ of HEU infants may only be functional. Conclusively, impaired neutrophil function could be a factor of vulnerability in HEU infants.

Keywords: HIV, neutrophils, reactive oxygen species, nitroblue tetrazolium, infants

\section{INTRODUCTION}

The implementation of mother-to-child transmission prevention programs worldwide is arguably one of the major public health successes of the present century. Indeed, the proposed recommendations on the antiretroviral therapy on mothers and their infants, together with improved obstetric management and the updated protocols on breastfeeding from HIV-infected mothers have considerably reduced the number of infected infants in developing countries $(1,2)$.

Currently, it is estimated that HIV-exposed uninfected (HEU) infants represent up to $30 \%$ of all births in Africa $(3,4)$. Although mother-to-child transmission prevention programs have dramatically reduced the mother-to-child transmission rate, HEU infants are still more vulnerable to infections when compared to HIV-unexposed (HU) infants from HIV-uninfected mothers. This was robustly demonstrated in a study conducted in Zimbabwe between 1987 and 2000, where more than 14,000 participants were enrolled (5). In this study, an elevated mortality rate of HEU infants was observed (5). The 2-year mortality rate was three times higher in the HEU cohort when compared to the HU cohort $(5,6)$. Morbidity and mortality were at their peak levels in the 3-6 months old children, and the majority of the deaths were associated to lower respiratory tract infections. This seminal observation has been supported by several other studies in Africa that have independently 
reported a more vulnerable immune system thus a higher risk of infection of HEU infants when compared to HU infants (7-13).

Intensive research on HIV and antiretroviral (ARV)-drug therapy has improved our understanding of the immune susceptibility of HIV-infected population (14-16); however, our understanding of HIV/therapy exposure on HEU infants is still limited. A number of studies have reported a lower numbers of naive $\mathrm{CD} 4^{+}$cells, a reduced thymic output (17) and an impaired humoral response to vaccines (18) in HEU infants. However, the vulnerability of the immune system of HEU infants in the context of the alteration of the production of reactive oxygen species (ROS), a class of signaling molecules that regulate many signaltransduction pathways and play critical roles in cell survival, death, and immune defenses during infection $(15,19)$ has never been addressed.

Neutrophils are major producers of ROS and essential effector cells of the innate immune response against invading pathogens and the pivots of innate immunity; as they are first and powerful defense system against invading pathogens (20). Microbicides and cytotoxic activities of neutrophils depend on very intricate mechanisms including the release of proteolytic enzymes and the rapid production of ROS (oxidative burst) (21). The respiratory burst is the major mechanism by which neutrophils kill engulfed bacteria (22). In the light of the foregoing and of the observed susceptibility of HEU infants to infections, we investigated the phagocytes oxidative capacity of HEU infants.

\section{MATERIALS AND METHODS}

\section{Participants}

Twenty-five healthy HEU infants aged 6- to 12-week olds visiting the National Laboratory of Public Health in Libreville (Gabon) were prospectively recruited from March to August 2015. The mothers and infants information on preventive treatment, breastfeeding, time of anti-viral therapy initiation were recorded.

HIV perinatal infection (RT-PCR Cobas, Biomerieux, France) were checked from each HEU infant in peripheral blood at 1.5, 3,6 , and 9 months after birth as part of their scheduled checked up in the reference laboratory in Gabon. An additional sample was taken to determine anti-HIV-1 antibodies seroconversion by ELISA at 18 months old. All HIV-positive infants and infants with any clinical condition at the time of recruitment were excluded from the study.

\section{Ethics Statement}

Informed consent was obtained from parents of all study participants. The National Laboratory of Public Health Ethics Review Board of Gabon approved the protocol. We adhered to the World Medical Association's Declaration of Helsinki and Good Clinical Practice guidelines during the treatment of all participants and handling of their personal data.

\section{Neutrophils Oxidative Burst Response Assay}

The neutrophils oxidative burst response was performed on blood collected from 6- or 12-week-old infants using the In vitro diagnostic quality Sigma-Aldrich nitroblue tetrazolium (NBT) reduction test (St. Louis, MO, USA). The test was done according to the manufacturer protocol. Briefly, venous blood from infants was drawn into a heparin tube. Heparinized blood $(0.05-0.2 \mathrm{ml})$ was mixed with the NBT mixture (1-1.2 ml) and $5 \mu$ l of stimulant (bacterial extracts/endotoxins) in the stimulated samples. Fifty microliters of heparinized blood-NBT mixture were transferred onto a clean glass slide to make a moderately thick smear. The smears were treated with $1 \mathrm{ml}$ of ACCUSTAIN Wright Stain for $15 \mathrm{~s}$ and flooded with $1 \mathrm{ml}$ of distilled water for $30 \mathrm{~s}$, rinsed and air-dried. Three readers evaluated the percentage of formazan-containing neutrophils on stained slides under a microscope.

\section{Interpretation NBT Reduction Test Results}

According to the literature, $69-100 \%$ of resting neutrophils are NBT-positive in healthy newborn infants (23). In the present study, we set the threshold for the diagnosis of oxidative burst defect at $60 \%$ of neutrophils reducing NBT after bacterial extracts stimulation. Therefore, neutrophils function was defined as normal if more than $60 \%$ of neutrophils reduced NBT after bacterial extracts stimulation. Impaired or reduced ROS production was evoked when a subject shows less than $60 \%$ of neutrophils reducing NBT after bacterial extracts stimulation.

\section{Statistical Analysis}

All statistical analyses were done using the software GraphPad Prism version 6. Descriptive statistics and odds ratios were used to characterize the study population. The difference between infants with neutrophils dysfunction and infants with functional neutrophils was assessed using the Mann-Whitney $U$-test (significance set $p$-value $\leq 0.05$ ).

\section{RESULTS AND DISCUSSION}

Infections in infants are an important cause of morbidity and mortality. Understanding their immune system is crucial to develop new approaches to fight and prevent diseases and improve their health. Because in their early life, infants depend mainly on their innate immune system for protection against infections, we studied HEU infants' neutrophils function.

Neutrophils oxidative burst was assessed for $25 \mathrm{HIV}$-exposed infants referred to our study site; The National Laboratory of Public Health (Libreville, Gabon). This study focused in HEU infants as the HU infants are not follow-up in our study site. It was therefore difficult to include HU children in this pilot study. Nevertheless, we manage to include three HU infants in the study. Their results are included in the study as technical control. The limited number of HU (3 HU infants) obtained in this study was not enough for experimental control. Their immune responses were not directly compared to HEU immune response. ROS are crucially involved in microbial killing $(22,24)$. Figure 1 illustrates the quantification of NBT-test negative and positive neutrophils. Seventeen of the twenty-five infants were males and eight were females as shown in the Table 1. Sixteen 


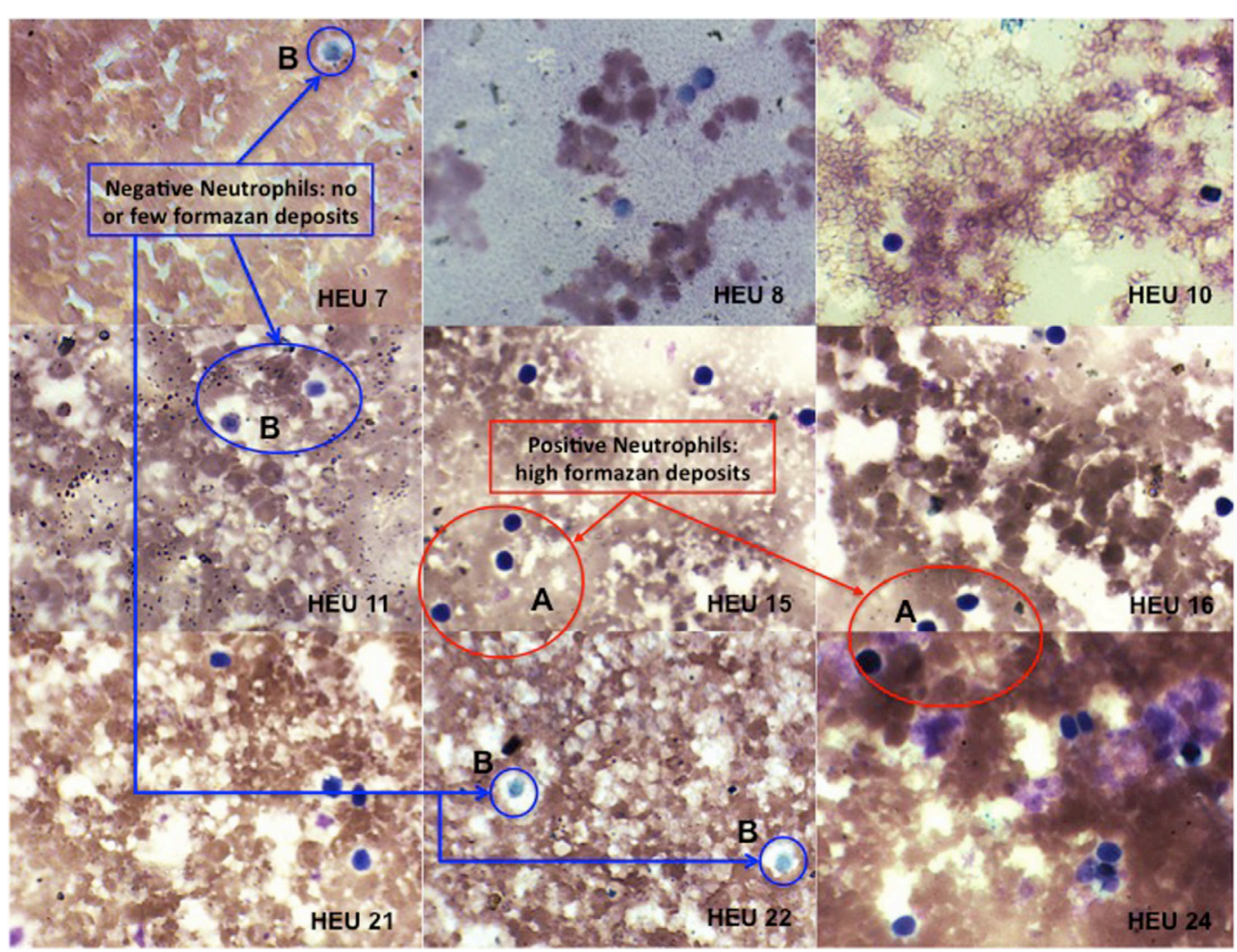

FIGURE 1 | Photomicrographs of nitroblue tetrazolium (NBT) dye reduction by blood neutrophils from HEU infants (at $\times 100$ magnification). Positive $=$ neutrophils contain increased formazan deposits visible as a large dark purple to black intra-cytoplasmic inclusions $(\mathbf{A})$. Negative $=$ neutrophils contain few or no formazan deposits (B).

HIV-exposed infants (64\%) had normal neutrophils profile after antigen stimulation. In those infants, the rate of neutrophils positive for formazan deposits (reduced NBT) after bacterial extracts stimulation ranged from 67 to $97.7 \%$ (Table 1). We observed a high number of resting neutrophils producing ROS (reducing NBT) in most HEU infants with functioning neutrophils. This observation was not surprising, as an increased reduction of NBT by neutrophils of newborn infants has already been reported $(23,25)$. Tovo and Ponzone illustrated the presence of humoral factors stimulating ROS secretion by neutrophils leading to positive NBT reduction (25) in the plasma of neonates. The high percentages of NBT positive cells could also be explained by in utero exposure to a pro-inflammatory fetal environment (26). Figure 2 shows selected HEU infants ROS secretion profile after antigen stimulation.

Nine HIV-exposed infants (36\%) showed impaired ROS production after stimulation with bacterial extracts (Table 1). In those infants, the rate of neutrophils positive for formazan deposits after bacterial extracts stimulation ranged from 3 to $35 \%$. The ability of neutrophil to produce ROS after exposure to bacteria or bacteria extracts indicates the functionality of these immune cells. Several studies have addressed the alteration of the neutrophil compartment in HEU infants. A telling example is that of an European collaborative study which reported that exposure to ARV does not affects HEU children neutrophils count during the first months of life (27). This is consistent with our present data and further reinforces the notion that neutrophil counts might not be a reliable early indicator of the defective immune system of HEU infants.

Our study does not only assess the number of neutrophils but beyond the quantitative appraisal of these important innate immune effector cells, also looks at their functionality in the form of ROS secretion. We report an impaired production of ROS by neutrophils of $36 \%$ of HEU infants suggestive of an impaired immune system in these infants. This finding is clearly important but would urgently require to be validated in a larger study. Given the small number, the primary results presented in this study could be a call to the scientific community to further investigate early marker(s) of immune dysfunction in HEU infants.

The metabolic defect observed in a number of HEU infants could find its explanations by the transfer of immunosuppressive viral products across the placenta that has been suggested to suppress infants' immune system without productive infection (4). Moreover, antenatal ARV-prophylaxis therapy for the prevention of mother-to-child transmission has been reported to 
TABLE 1 | Nitroblue tetrazolium (NBT)-test results and clinical information.

\begin{tabular}{|c|c|c|c|c|c|c|c|c|c|c|}
\hline \multirow[t]{2}{*}{ Infant ID } & \multirow{2}{*}{$\begin{array}{l}\text { Age (in } \\
\text { weeks) }\end{array}$} & \multirow[t]{2}{*}{ Gender } & \multicolumn{2}{|c|}{ NBT-positive neutrophils } & \multirow[t]{2}{*}{ Interpretation } & \multirow[t]{2}{*}{ Infants preventive Thx } & \multirow[t]{2}{*}{ Breastfeeding } & \multicolumn{3}{|c|}{ Mother start of antiretroviral therapy } \\
\hline & & & $\begin{array}{c}\text { Non- } \\
\text { stimulated } \\
(\%)\end{array}$ & $\begin{array}{l}\text { Stimulated } \\
(\%)\end{array}$ & & & & $\begin{array}{l}\text { Before } \\
\text { pregnancy }\end{array}$ & During pregnancy & Therapy \\
\hline HEU1 & 10 & Male & 86.7 & 88 & Normal neutrophils & Nevirapine/co-trimoxazole & No & No & Yes@ 6 months & EFV-TDF-LVD \\
\hline HEU2 & 6 & Male & 60 & 89 & Normal neutrophils & Nevirapine/co-trimoxazole & No & Yes & No & TDF-3TC-EFV \\
\hline HEU3 & 12 & Female & 75 & 85 & Normal neutrophils & Zidovidine/co-trimoxazole & No & Yes & No & 3TC-Zido-EFV \\
\hline HEU4 & 6 & Female & 73 & 85 & Normal neutrophils & & & Yes & No & TDF-3TC-EFV \\
\hline HEU5 & 12 & Female & 47 & 92.7 & Normal neutrophils & Zidovidine/co-trimoxazole & No & Yes & No & \\
\hline HEU6 & 6 & Male & 78 & 91 & Normal neutrophils & Nevirapine/co-trimoxazole & No & Yes & No & \\
\hline HEU7 & 6 & Female & 9.3 & 12.7 & $\begin{array}{l}\text { Impaired reactive oxygen species } \\
\text { (ROS) production }\end{array}$ & Nevirapine/co-trimoxazole & No & No & No & None \\
\hline HEU8 & 10 & Male & 8.7 & 4.7 & Impaired ROS production & Nevirapine/co-trimoxazole & No & No & Yes@ 4 months & TDF-FTC-EFV \\
\hline HEU9 & 10 & Male & 9.5 & 7 & Impaired ROS production & Nevirapine/co-trimoxazole & No & No & Yes@ 4 months & TDF-FTC-EFV \\
\hline HEU10 & 6 & Male & 14 & 35 & Impaired ROS production & Nevirapine & No & Yes & NO & \\
\hline HEU11 & 6 & Female & 48.3 & 38 & Impaired ROS production & Nevirapine/co-trimoxazole & No & No & Yes@ 6 months & \\
\hline HEU12 & 6 & Male & 37 & 34 & Impaired ROS production & Nevirapine/co-trimoxazole & No & No & Yes @ 2 months & \\
\hline HEU13 & 6 & Female & 6.3 & 97.7 & Normal neutrophils & Nevirapine/co-trimoxazole & No & Yes & & $\begin{array}{l}\text { ABC-3TC-LPV- } \\
\text { RTV }\end{array}$ \\
\hline HEU14 & 12 & Male & 14 & 23 & Impaired ROS production & Nevirapine/co-trimoxazole & $\begin{array}{l}\text { Yes (for } \\
3 \text { months) }\end{array}$ & No & Yes@ 6 months & TDF-3TC-EFV \\
\hline HEU15 & 6 & Male & 93.7 & 94.7 & Normal neutrophils & NO & Yes & No & Yes@ 3 months & TDF-FTC-EFV \\
\hline HEU16 & 6 & Male & 91.7 & 98 & Normal neutrophils & Nevirapine/co-trimoxazole & No & Yes & No & \\
\hline HEU17 & 8 & Male & 96 & 99 & Normal neutrophils & Nevirapine & No & No & Yes@ 3 months & TDF-FTC-EFV \\
\hline HEU18 & 7 & Male & 75 & 81.3 & Normal neutrophils & Nevirapine/co-trimoxazole & No & & Yes@ 4 months & \\
\hline HEU19 & 6 & Female & 74.7 & 73.3 & Normal neutrophils & Nevirapine/co-trimoxazole & No & No & Yes@ 4 months & \\
\hline HEU2O & 6 & Male & 86 & 88 & Normal neutrophils & Nevirapine/co-trimoxazole & No & Yes & No & \\
\hline HEU21 & 6 & Female & 33.3 & 30.5 & Impaired ROS production & Nevirapine/co-trimoxazole & No & Yes & No & \\
\hline HEU22 & 6 & Male & 3 & 3 & Impaired ROS production & Nevirapine/co-trimoxazole & No & Yes & No & TDF-3TC-EFV \\
\hline HEU23 & 6 & Male & 74.7 & 67 & Normal neutrophils & Nevirapine/co-trimoxazole & No & Yes & No & \\
\hline HEU24 & 6 & Male & 67 & 80 & Normal neutrophils & Nevirapine/co-trimoxazole & No & Yes & No & AZT-3TC-NVP \\
\hline HEU25 & 6 & Male & 74 & 83.7 & Normal neutrophils & Nevirapine/co-trimoxazole & No & No & Yes@ 7 months & AZT-3TC-NVP \\
\hline HU1 & 12 & Female & 56 & 72.7 & Normal neutrophils & NA & NA & NA & NA & NA \\
\hline HU2 & 8 & Female & 53 & 92 & Normal neutrophils & NA & NA & NA & NA & NA \\
\hline HU3 & 6 & Male & 76.3 & 95.3 & Normal neutrophils & NA & NA & NA & NA & NA \\
\hline
\end{tabular}

Bold font indicates Impaired ROS production. 


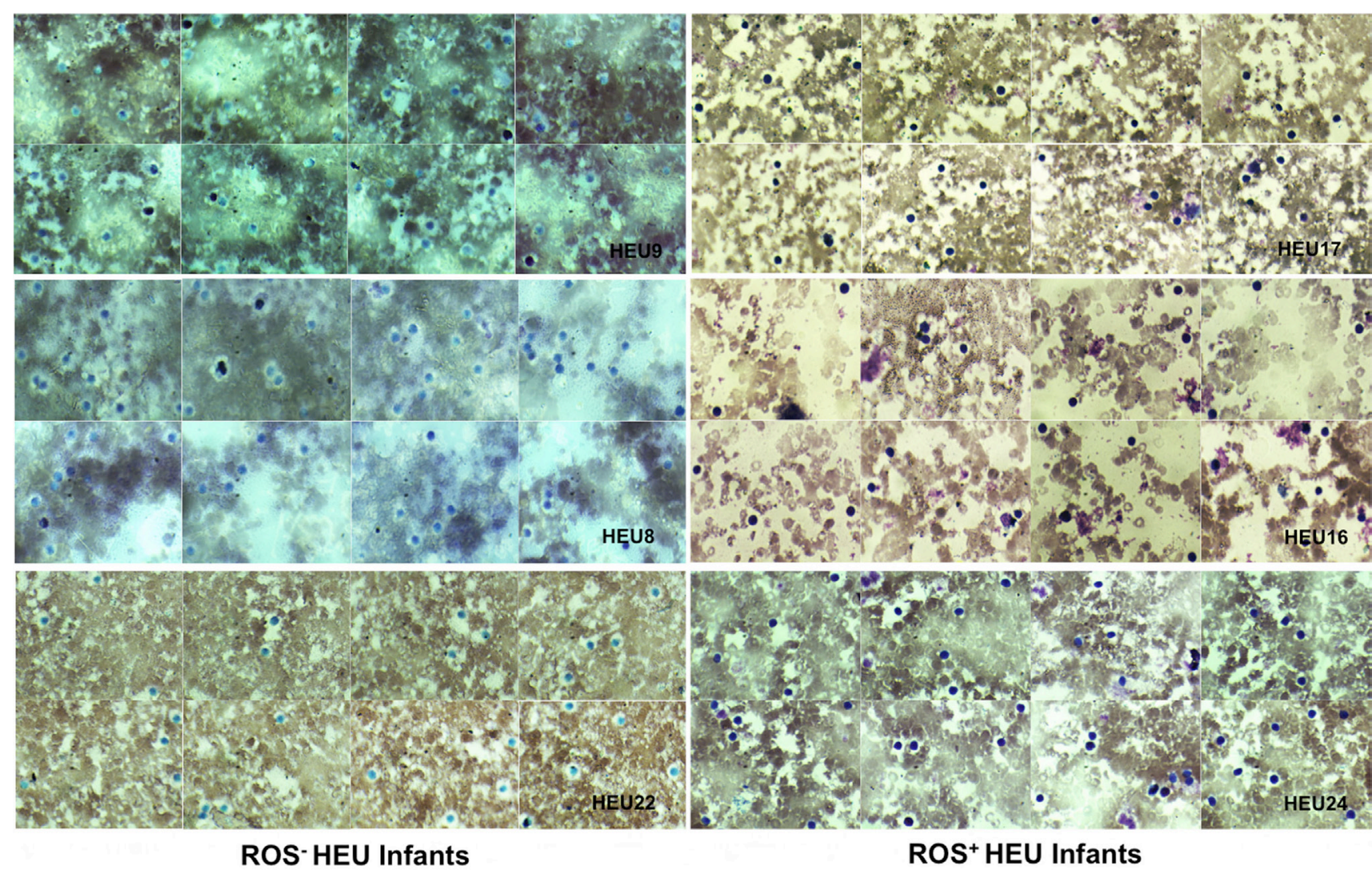

FIGURE 2 | Photomicrographs of nitroblue tetrazolium (NBT) dye reduction profiles of selected HIV-exposed uninfected (HEU) infants blood neutrophils (at $\times 100$ magnification). Reactive oxygen species (ROS)- HEU infants indicate infants with impaired ROS production. ROS ${ }^{+} \mathrm{HEU}$ infants indicate infants with normal ROS production. ROS + HEU infants illustrated by HEU 17, 16, and 27 showed increased formazan deposits visible as a large dark purple to black intra-cytoplasmic inclusions whereas, ROS- HEU infants illustrated by HEU 8, 9, and 22 showed few or no formazan deposits.

be toxic to mitochondria and to modulate the immune system of HEU infants (4). This was further suggested experimentally by Michailidis et al., who showed that neutrophil oxidative burst was significantly lower in HIV-patients receiving highly active anti-retroviral treatment (HAART) when compared to treatment-naïve patients, supporting the plausible link between HAART and neutrophils impaired oxidative burst (28). We now provide additional evidences for such a link as our contingency table analysis showed that infants born from mothers who started ARV-therapy during pregnancy had an odds ratio of 2.8 for oxidative burst defect when compared to infants born from mothers who started ARV-therapy before pregnancy. Although suggestive, our present findings need to be validated in larger studies for dispositive answers on the role of ARV and/or HIV in utero HIV exposure on the neutrophil functions and the relationship of this apparent interdependence on the impaired immune system of HEU infants.

We believe that the observed impaired function of neutrophils in HEU infants to be transient. In fact, a couple of HEU infants who had impaired neutrophils function and that was brought for an unscheduled visit 2 months after the recruitment had recovered neutrophils function (data not shown). This is not surprising as Le Chenadec et al. reported that ARV exposure had a transient effect on several selected hematological variables in HEU infants (29).

Comparing the blood of infants with dysfunction neutrophil function with that of infants with unaltered responsiveness, we found no significant differences in their total numbers of neutrophils, lymphocytes, and leukocytes (Figure 3) further re-emphasizing the poor potential of assessing total immune cell numbers to predict the vulnerability of the immune system of HEU infants.

\section{CONCLUSION}

The present study contributes to the global effort to understand the immune determinants of HEU infants increased susceptibility to infections during the first month of their life. Using NBT reduction assay, we found impaired oxidative burst in $36 \%$ of HEU infants evaluated with no alteration in neutrophil counts suggesting that impaired neutrophils function could be one of the immune dysfunctions found in a number of 

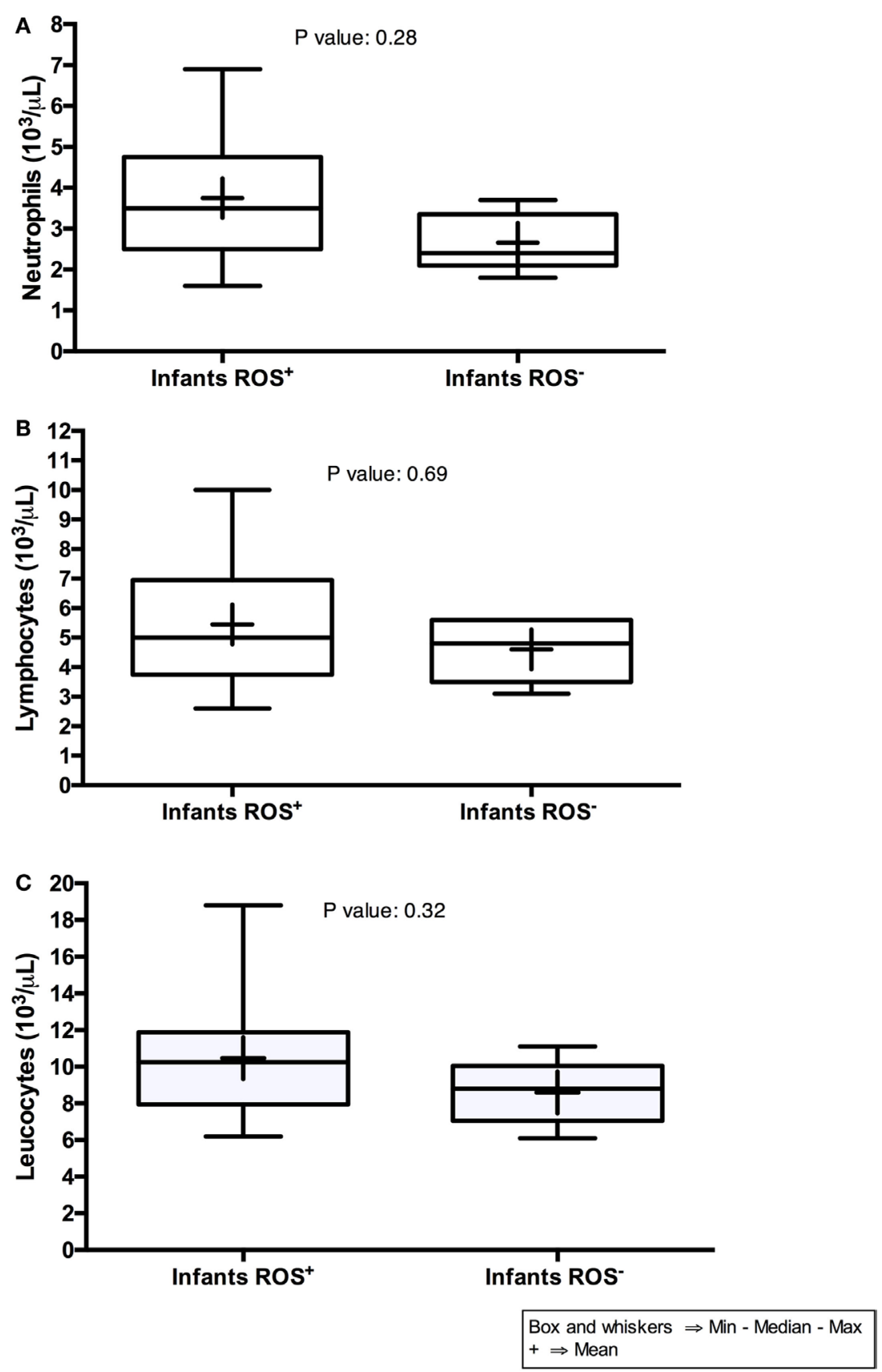

FIGURE 3 | Neutrophils, lymphocytes, and leukocytes counts of HIV-exposed uninfected infants. On the graphs, Infants (ROS ${ }^{+}$) indicate infants with normal reactive oxygen species (ROS) production, whereas Infants $\left(R O S^{-}\right)$indicate infants with impaired ROS production.

HEU infants. The present results need to be validated in a larger study.

\section{AUTHOR CONTRIBUTIONS}

AS, AM-N, and ON: recruited participant, performed research, and analyzed data. AN: contributed to provide analytical tools. PE: designed research and proofread the paper. JS: designed the research, performed research, contributed vital reagents and analytical tools, analyzed data, and wrote the paper.

\section{ACKNOWLEDGMENTS}

We are grateful to all mothers who gave their consent and participated in the study. Thanks to the Pasteur Network, many thanks to Dr. Margarita Hurtado-Nedelec (MD, PhD) and the 
Department of Hematology and Immunology Immune, UF of Immune Dysfunctions of the University Hospital Bichat-Claude Bernard in Paris (France) for training our Biologists. Dr. Marie José Stasia-Pauger (PharmD, PhD, HDR) from Grenoble University Hospital, France. Also we would like to acknowledge Dr. Justin Komguep Nono from Institute of Infectious Diseases and

\section{REFERENCES}

1. Tonwe-Gold B, Ekouevi DK, Viho I, Amani-Bosse C, Toure S, Coffie PA, et al. Antiretroviral treatment and prevention of peripartum and postnatal HIV transmission in West Africa: evaluation of a two-tiered approach. PLoS Med (2007) 4(8):e257. doi:10.1371/journal.pmed.0040257

2. Siegfried N, van der Merwe L, Brocklehurst P, Sint TT. Antiretrovirals for reducing the risk of mother-to-child transmission of HIV infection. Cochrane Database Syst Rev (2011) (7):CD003510. doi:10.1002/14651858.CD003510. pub3

3. Shapiro RL, Lockman S. Mortality among HIV-exposed infants: the first and final frontier. Clin Infect Dis (2010) 50(3):445-7. doi:10.1086/649887

4. Afran L, Garcia Knight M, Nduati E, Urban BC, Heyderman RS, RowlandJones SL. HIV-exposed uninfected children: a growing population with a vulnerable immune system? Clin Exp Immunol (2014) 176(1):11-22. doi:10.1111/ cei. 12251

5. Marinda E, Humphrey JH, Iliff PJ, Mutasa K, Nathoo KJ, Piwoz EG, et al. Child mortality according to maternal and infant HIV status in Zimbabwe. Pediatr Infect Dis J (2007) 26(6):519-26. doi:10.1097/01.inf.0000264527. 69954.4c

6. Newell ML, Brahmbhatt H, Ghys PD. Child mortality and HIV infection in Africa: a review. AIDS (2004) 18(Suppl 2):S27-34. doi:10.1097/00002030200406002-00004

7. Brahmbhatt H, Kigozi G, Wabwire-Mangen F, Serwadda D, Lutalo T, Nalugoda F, et al. Mortality in HIV-infected and uninfected children of HIV-infected and uninfected mothers in rural Uganda. J Acquir Immune Defic Syndr (2006) 41(4):504-8. doi:10.1097/01.qai.0000188122.15493.0a

8. Landes M, van Lettow M, Bedell R, Mayuni I, Chan AK, Tenthani L, et al. Mortality and health outcomes in HIV-infected and HIV-uninfected mothers at 18-20 months postpartum in Zomba District, Malawi. PLoS One (2012) 7(9):e44396. doi:10.1371/journal.pone.0044396

9. Landes M, van Lettow M, Chan AK, Mayuni I, Schouten EJ, Bedell RA. Mortality and health outcomes of HIV-exposed and unexposed children in a PMTCT cohort in Malawi. PLoS One (2012) 7(10):e47337. doi:10.1371/ journal.pone.0047337

10. Fawzy A, Arpadi S, Kankasa C, Sinkala M, Mwiya M, Thea DM, et al. Early weaning increases diarrhea morbidity and mortality among uninfected children born to HIV-infected mothers in Zambia. J Infect Dis (2011) 203(9):1222-30. doi:10.1093/infdis/jir019

11. Slogrove A, Reikie B, Naidoo S, De Beer C, Ho K, Cotton M, et al. HIVexposed uninfected infants are at increased risk for severe infections in the first year of life. J Trop Pediatr (2012) 58(6):505-8. doi:10.1093/tropej/ fms019

12. Slogrove AL, Cotton MF, Esser MM. Severe infections in HIV-exposed uninfected infants: clinical evidence of immunodeficiency. J Trop Pediatr (2010) 56(2):75-81. doi:10.1093/tropej/fmp057

13. Liu L, Oza S, Hogan D, Perin J, Rudan I, Lawn JE, et al. Global, regional, and national causes of child mortality in 2000-13, with projections to inform post-2015 priorities: an updated systematic analysis. Lancet (2015) 385(9966):430-40. doi:10.1016/S0140-6736(14)61698-6

14. Hayani KC, Verral SC, Pitrak DL. Impaired phagocyte oxidative capacity in human immunodeficiency virus-infected children. J Infect Dis (1999) 179(3):584-9. doi:10.1086/314622

15. Bowers NL, Helton ES, Huijbregts RP, Goepfert PA, Heath SL, Hel Z. Immune suppression by neutrophils in HIV-1 infection: role of PD-L1/PD-1 pathway. PLoS Pathog (2014) 10(3):e1003993. doi:10.1371/journal.ppat.1003993
Molecular Medicine, Division of Immunology of the University of Cape Town for the for critical reading of the manuscript.

\section{FUNDING}

This study was entirely funded by the Gabonese Government.

16. Pittis MG, Prada F, Mangano A, Perez L, Sternik G, Redondo J, et al. Monocyte phagolysosomal fusion in children born to human immunodeficiency virus-infected mothers. Pediatr Infect Dis J (1997) 16(1):24-8. doi:10.1097/00006454-199701000-00006

17. Nielsen SD, Jeppesen DL, Kolte L, Clark DR, Sorensen TU, Dreves AM, et al. Impaired progenitor cell function in HIV-negative infants of HIV-positive mothers results in decreased thymic output and low CD4 counts. Blood (2001) 98(2):398-404. doi:10.1182/blood.V98.2.398

18. Abramczuk BM, Mazzola TN, Moreno YM, Zorzeto TQ, Quintilio W, Wolf PS, et al. Impaired humoral response to vaccines among HIV-exposed uninfected infants. Clin Vaccine Immunol (2011) 18(9):1406-9. doi:10.1128/ CVI.05065-11

19. Schieber M, Chandel NS. ROS function in redox signaling and oxidative stress. Curr Biol (2014) 24(10):R453-62. doi:10.1016/j.cub.2014.03.034

20. Mantovani A, Cassatella MA, Costantini C, Jaillon S. Neutrophils in the activation and regulation of innate and adaptive immunity. Nat Rev Immunol (2011) 11(8):519-31. doi:10.1038/nri3024

21. Nauseef WM, Borregaard N. Neutrophils at work. Nat Immunol (2014) 15(7):602-11. doi:10.1038/ni.2921

22. Segal AW. How neutrophils kill microbes. Annu Rev Immunol (2005) 23:197-223. doi:10.1146/annurev.immunol.23.021704.115653

23. Humbert JR, Kurtz ML, Hathaway WE. Increased reduction of nitrobule tetrazolium by neutrophils of newborn infants. Pediatrics (1970) 45(1):125-8.

24. Urban CF, Lourido S, Zychlinsky A. How do microbes evade neutrophil killing? Cell Microbiol (2006) 8(11):1687-96. doi:10.1111/j.1462-5822. 2006.00792.x

25. Tovo PA, Ponzone A. Cellular and humoral factors involvement in the enhanced NBT reduction by neutrophil leucocytes of newborn infants. Acta Paediatr Scand (1977) 66(5):549-52. doi:10.1111/j.1651-2227.1977. tb07945.x

26. Evans C, Jones CE, Prendergast AJ. HIV-exposed, uninfected infants: new global challenges in the era of paediatric HIV elimination. Lancet Infect Dis (2016) 16(6):e92-107. doi:10.1016/S1473-3099(16)00055-4

27. European Collaborative S. Levels and patterns of neutrophil cell counts over the first 8 years of life in children of HIV-1-infected mothers. AIDS (2004) 18(15):2009-17. doi:10.1097/00002030-200410210-00005

28. Michailidis C, Giannopoulos G, Vigklis V, Armenis K, Tsakris A, Gargalianos P. Impaired phagocytosis among patients infected by the human immunodeficiency virus: implication for a role of highly active anti-retroviral therapy. Clin Exp Immunol (2012) 167(3):499-504. doi:10.1111/j.1365-2249.2011. 04526.x

29. Le Chenadec J, Mayaux MJ, Guihenneuc-Jouyaux C, Blanche S; Enquete Perinatale Francaise Study Group. Perinatal antiretroviral treatment and hematopoiesis in HIV-uninfected infants. AIDS (2003) 17(14):2053-61. doi:10.1097/01.aids.0000076321.42412.b4

Conflict of Interest Statement: The authors declare that they have no competing interests. Also no financial or other competing interests exist.

Copyright (C) 2017 Maloupazoa Siawaya, Mveang-Nzoghe, Mvoundza Ndjindji, Mintsa Ndong, Essone and Djoba Siawaya. This is an open-access article distributed under the terms of the Creative Commons Attribution License (CC BY). The use, distribution or reproduction in other forums is permitted, provided the original author(s) or licensor are credited and that the original publication in this journal is cited, in accordance with accepted academic practice. No use, distribution or reproduction is permitted which does not comply with these terms. 